\title{
Analysis of YAP1 and TAZ expression by immunohistochemical staining in malignant mesothelioma and reactive mesothelial cells
}

\author{
YUSUKE TAKEHARA, TOSHIKO YAMOCHI, TASUKU NAGUMO, TOMONARI CHO, FUMIHIKO URUSHIBARA, \\ KOHEI ONO, TOMONORI FUJII, NAOKO OKAMOTO, YOSUKE SASAKI, SAKIKO TAZAWA, MAYUMI HONMA, \\ TOMOKO NOROSE, EISUKE SHIOZAWA, GENSHU TATE and MASAFUMI TAKIMOTO
}

Department of Pathology and Laboratory Medicine, Showa University School of Medicine, Tokyo 142-8555, Japan

Received November 2, 2016; Accepted March 7, 2017

DOI: $10.3892 / 01.2018 .8225$

Abstract. Gene mutations are involved in the development of malignant mesothelioma. Important mutations have been identified in the genes for cyclin-dependent kinase inhibitor 2A (p16) alternative reading frame, breast cancer-associated protein $1(B A P l)$ and neurofibromatosis type 2 (NF2). Previously, the utility of detecting the loss of $B A P 1$ by immunohistochemistry (IHC) and p16-deletion by fluorescence in situ hybridization has been identified in seyeral studies. However, NF2-associated examinations have not been performed. The present study aimed to evaluate the expression of yes-associated protein 1 (YAP1) and tafazzin (TAZ) protein, which are associated with NF2 gene mutations, in malignant mesothelioma (MM) and reactive mesothelial cells (RMCs). Formalin-fixed paraffin-embedded tisstes from $31 \mathrm{MM}$ and 33 RMC samples were analyzed. The expression of YAP1 and TAZ protein were examined by IHC. Positivity for YAP1 was identified 27/31 MM and 15/33 RMC samples. Positivity for TAZ was identified in 28/31 MM and 18/33 RMC samples. Using the optimal cutoff points determined by the receiver

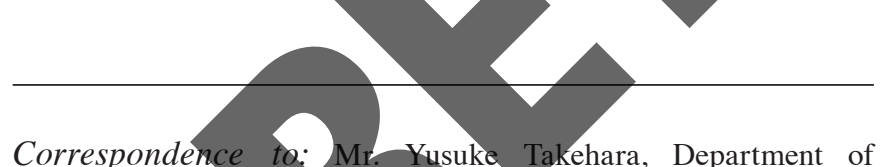
Pathology and Laboratory Medicine, Showa University School of Medicine, 1-5-8 Hatanodai, Shinagawa, Tokyo 142-8555, Japan E-mail: yuutake47@yahoo.co.jp

Abbreviations: BAP1, breast cancer-associated protein 1; FFPE, formalin-fixed paraffin-embedded; FISH, fluorescence in situ hybridization; IHC, immunohistochemistry; Merlin, moesin-ezrin-radixin-like protein; MM, malignant mesothelioma; $\mathrm{NF}$ 2, neurofibromatosis type 2; RMC, reactive mesothelial cell; TAZ, tafazzin; YAP1, yes-associated protein 1; CD44, cluster of differentiation 44; LATS2, large tumor suppressor 2; TEAD, TEA domain; PP1, phosphoprotein 1; ASPP2, apoptosis-stimulating of p53 protein 2; Amot, angiomotin; ROC, receiver operating characteristic; AUC, area under curve

Key words: malignant mesothelioma, neurofibromatosis type 2, Hippo pathway, yes-associated protein 1, tafazzin operating characteristic curve, a positive IHC result for YAP1 and TAZ was $74 \%$ sensitive and $94 \%$ specific for detecting MM. The results indicate that increased expression of YAP1 and TAZ may be associated with mesothelial tumorization, and aid-in the diagnosis of MM.

\section{Introduction}

Malignant mesothelioma (MM) is an aggressive tumor that develops from the pleura or other mesothelial surfaces and is frequently associated with previous exposure to asbestos. The diagnosis of MM is based primarily on histopathological features, and immunohistochemistry (IHC) is used to provide additional support for the diagnosis of MM. However, MM may be classified as epithelioid, biphasic or sarcomatoid type, and it can therefore be difficult to diagnose as the histological subtypes exhibit different staining patterns (1). At present, concerning the development of MM, important mutations have been identified in the genes for cyclin-dependent kinase inhibitor 2A (p16) alternative reading frame, breast cancer-associated protein $1(B A P 1)$, and neurofibromatosis type 2 (NF2) $(2,3)$. These genes serve as tumor suppressor genes, and have been demonstrated to be inactivated in patients with MM (2,3). Previous studies suggest that detection of 9p21 homozygous deletion using fluorescence in situ hybridization (FISH) and loss of BAPI by IHC analysis is useful for diagnosing MM (4,5). However, NF2-related FISH and/or IHC analyses for diagnosing MM have not been adequately discussed. The NF2 gene is on chromosome $22 \mathrm{q} 12$, and encodes a tumor suppressor protein, moesin-ezrin-radixin-like protein (Merlin), which is a cytoskeletal linker protein (6). Merlin is regulated by extracellular signaling such as that by cluster of differentiation (CD)44 and adherens junctions $(2,6)$. Merlin modulates multiple cellular signal transduction cascades, such as the mechanistic target of rapamycin pathway and the Hippo signaling pathway $(2,3,6)$. The Hippo signaling pathway regulates organ size, development and differentiation, and tissue regeneration by restricting cell growth, regulating cell division and promoting apoptosis $(3,6)$. The four core components in the Hippo pathway are macrophage-stimulating protein $1 / 2$, Salvador 1, Mps one binder 1 and large tumor 
suppressor 1/2 (LATS1/2), all of which act as tumor suppressors. Subsequent to receiving upstream signals, for example from Merlin, the transcriptional coactivators yes-associated protein 1 (YAP1) and tafazzin (TAZ) are inactivated. Hippo signaling inactivation leads to constitutive YAP1/TAZ activation. Overexpression of YAP1 and an inactivating mutation of LATS2 have been identified in MM $(7,8)$. The TEA domain family of transcription factors are activated by YAP1/TAZ. The activation of YAP1/TAZ induces the transcription of multiple tumor-promoting genes, including cyclin D1 and connective tissue growth factor (CTGF) $(2,6)$. The expression of CTGF is associated with the abundant extracellular matrix formation of MM tissue, particularly in sarcomatoid MM. Scientists have hypothesized that TAZ, which may be a homolog of YAP1, may have different effects $(2,9,10)$. TAZ phosphorylation is modulated by PP1A and its interacting protein ASPP2 (10). PP1 efficiently dephosphorylates Ser-89 and Ser-311 in TAZ in vitro. However, YAP dephosphorylation is not modulated by PP1A in the same way as with TAZ (10). Furthermore, TAZ has been demonstrated to be involved in the development of multiple organs, including the lungs and the heart, as well as in numerous cellular processes, including stem cell differentiation, cell proliferation, and epithelial-mesenchymal transition (10). These effects have not yet been demonstrated in YAP. In addition, changes in the localization of YAP1 and TAZ via binding angiomotin, ASPP2 and $\alpha$-catenin have been reported (2,9-12).

In the present study, the expression of YAP1 and TAZ were evaluated using IHC. In addition, markers of MM were examined, and it was investigated whether combining the IHC analysis of YAP1 and TAZ may aid in distinguishing MM from reactive mesothelial cells (RMC) in clinical specimens.

\section{Materials and methods}

Patient samples. The records MM (26 pleural and 5 peritoneal), and 33 cases of RMC were collected from the archives of the Department of Pathology and Laboratory Medicine at Showa University School of Medicine (Tokyo, Japan) between April 2004 and March 2014. For MM, 20 patients were diagnosed from surgical specimens, 1 patient from an autopsy specimen and 10 patients from a biopsy specimen. For RMC, all patients were diagnosed from surgical specimens. Included in the present study were 7 women and 24 men with MM, with an age range of 55-89 years (median age, 73 years); and 5 female patients and 28 male patients with RMC with an age range of 15-66 years (median age, 29 years). Formalin-fixed paraffin-embedded (FFPE) tissue blocks were available for all patients. The tumor diagnosis was defined and sub-classified histologically according to the World Health Organization guidelines (13). The diagnosis of MM was based on routine hematoxylin-eosin histology and confirmed by IHC using antibodies against calretinin, Wilms tumor 1, D2-40, cytokeratin (CK) AE1/AE3, CK CAM 5.2, carcinoembryonic antigen, thyroid transcription factor 1 , and epithelial cell adhesion molecule (Table I). IHC studies were performed using an autoimmunostainer (Histostainer 36; Nichirei Bioscience Inc., Tokyo, Japan). Sections were incubated with $3 \% \mathrm{H}_{2} \mathrm{O}_{2}$ solution at room temperature for $5 \mathrm{~min}$ to block endogenous peroxidase activity. The primary antibody was added to the sections and the sections were incubated at room temperature for $15 \mathrm{~min}$. Subsequently, the secondary antibody (Histofine SimpleStain MAX-PO MULTI; undiluted; catalogue no. 724152; Nichirei Bioscience Inc.) was added to the sections and the sections were incubated at room temperature for $15 \mathrm{~min}$. The histological subtypes were epithelioid in 18 patients, biphasic in 9 patients, and sarcomatoid (including the desmoplastic type) in 4 patients. Cases of RMC were diagnosed from surgically resected specimens of emphysematous bullae from patients without a history of malignant disease. Representative tissue blocks were selected for IHC analysis. None of the patients with RMC had developed MM at the termination of the present study (April 2016). Appropriate research ethics and review board permissions were obtained from the Department of Pathology and Laboratory Medicine at Showa University School of Medicine (Tokyo, Japan; approval no. 1928). Written, informed consent was obtained from all patients prior to inclusion.

IHC. Sections (3- $\mu \mathrm{m}$ thickness) were cut from FFPE blocks. Antibody information is shown in Table I.For YAP1, the slides were pretreated for $40 \mathrm{~min}$ in a steamer with $\mathrm{pH} 9$ Tris-EDTA buffer, and rabbit monoclonal anti-human YAP1 (dilution, 1:500) was ussed. For TAZ, rabbit polyclonal anti-human TAZ (dilution, 1:50) was used. IHC studies were performed using an autoimmunostainer (Leica Bond-III; Leica Biosystems, Buffalo Grove, IL, USA). IHC staining was performed using the BOND Polymer Refine Detection system kit (catalogue no. DS9800; Leica Biosystems). Sections were incubated in $3 \% \mathrm{H}_{2} \mathrm{O}_{2}$ solution at room temperature for $5 \mathrm{~min}$ to block endogenous peroxidase activity. For YAP1, sections were incubated with the primary antibody at $4^{\circ} \mathrm{C}$ overnight, followed by ncubation with the secondary antibody at room temperature $8 \mathrm{~min}$. For TAZ, sections were incubated with the primary antibody at room temperature for $8 \mathrm{~min}$ followed by incubation with the secondary antibody at room temperature for $8 \mathrm{~min}$.

Evaluation of IHC. IHC results for YAP1 showed negative (0), weak (1+), equal (2+), and stronger (3+) staining in the nucleus compared with that in the cytoplasm. A positive result for YAP1 was identified by equal or stronger staining in the nucleus compared with that in the cytoplasm (score, $2+$ or $3+$, respectively) (Fig. 1A-D) (7). A positive result for TAZ was identified by strong staining in the cell membrane (Fig. 2A and B) (14). A positive result for TAZ was scored 1+ and no staining was scored as 0 . A minimum of 100 cells were evaluated. Staining results were scored as the percentage of stained mesothelial or tumor cells in 5\% increments. When $>5 \%$ of the mesothelial or tumor cells appeared stained by an antibody, the result was defined as positive. The intensity score was defined as $2+$ and $3+$ for YAP1, and $1+$ for TAZ. The samples were scored based on the total percentage of positive cells $(\leq 5 \%$, score $0 ; 6-25 \%$, score $1 ; 26-50 \%$, score $2 ; 51-75 \%$, score 3 ; and $>75 \%$, score 4 ) and intensity of the staining (2+ or $3+$ for YAP1, and $1+$ for TAZ). The total score represents the positive percentage score multiplied by the intensity score.

Statistical analysis. Statistical analysis was performed using JMP version 11 (SAS Institute Inc., Cary, NC, USA). The $\chi^{2}$ test and Fisher's exact probability test (two-tailed) were used to compare pathological features between the MM group and 


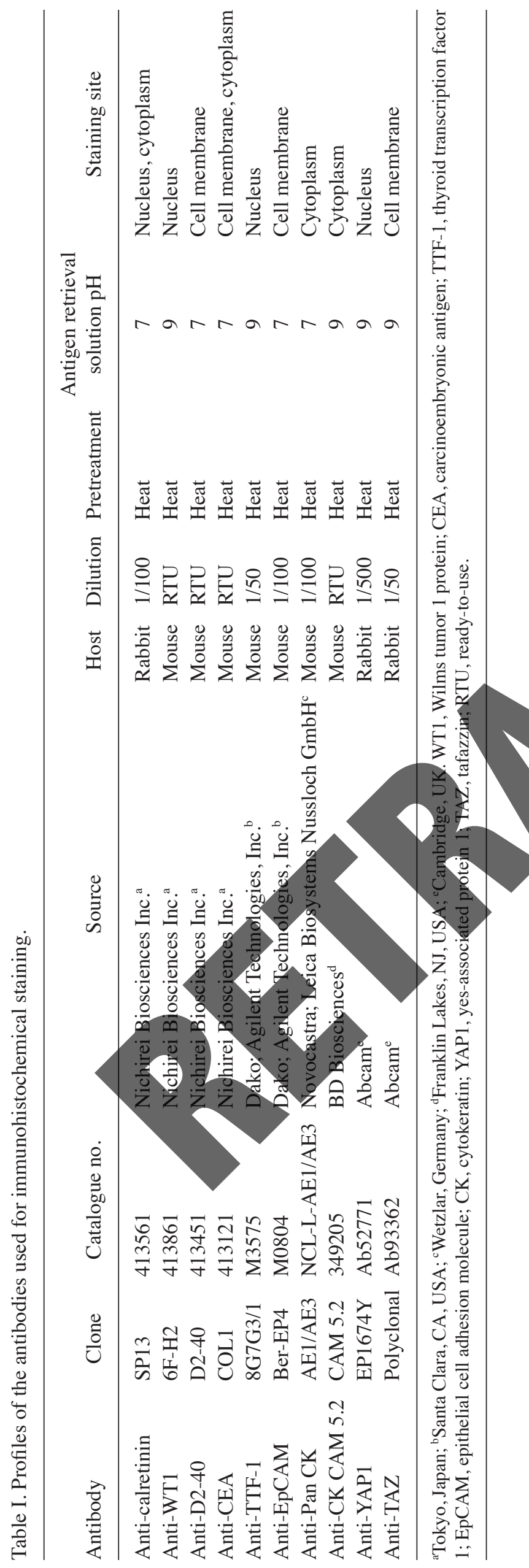

the RMC group. For all analyses, $\mathrm{P}<0.05$ was considered to indicate a statistically significant difference.

Receiver operating characteristic (ROC) curves were used to determine the association between the sensitivity and specificity of each antibody, and to find the optimal diagnostic cutoff values. The area under the ROC curve (AUC) was calculated and compared between each antibody.

Test characteristics were calculated for the individual markers and for certain markers in combination. Sensitivity [(true positives)/(true positives+false negatives)] and specificity [(true negatives)/(false positives+true negatives)] were determined, and their associated $95 \%$ confidence intervals (95\% CIs) were calculated by the following formula (n which ' $s$ ' is the sensitivity or specificity and ' $n$ ' is the total number of cases evaluated): $s \pm 1.96 \mathrm{x}$

\section{Results}

YAP1 and TAZ expression. Scores for the IHC analysis of YAP1 and TAZ were obtained for all patients. The results of IHC for MM and RMC are summarized in Table II.

A YAP1-positive result was determined for $27(87 \%)$ of 31 patients with MM, and $15(45 \%)$ of 33 patients with RMC; this difference between MM and RMC was statistically significant ( $\mathrm{P}=0.0006$; Fig. 3A). The mean total score was 9 in MM (range, 0-12), and 3 in RMC (range, 0-12).

A TAZ-positive result occurred in $28(90 \%)$ of 31 patients with MM, and $18(55 \%)$ of 33 patients with $\mathrm{RMC}(\mathrm{P}=0.0020$; Fig. 3B). The mean total score was 4 in MM, and 2 in RMC, with total scores ranging from 0 to 4 in both groups.

Diagnostic utility of YAPI and TAZ IHC analysis. ROC curves were constructed for YAP1 and TAZ to assess the ability of each marker to distinguish between MM and RMC. The AUC for YAP1 was 0.81 , while the AUC for TAZ was 0.77 . When the cutoff points for MM diagnosis were set at scores of $\geq 6$ for YAP1 and $\geq 3$ for TAZ (the optimal cutoff points determined by the ROC curve), the sensitivity and specificity values for these markers alone to distinguish MM from RMC were 84 and $79 \%$ for YAP1, and 87 and $61 \%$ for TAZ, respectively (Table III). These sensitivity and specificity values suggested that YAP1 or TAZ alone may not be useful for distinguishing $\mathrm{MM}$ from RMC in clinical practice. However, when considering the combination of YAP1 and TAZ using the same cutoff points, the sensitivity and specificity values were 74 and 94\% for distinguishing MM from RMC (Table III). Thus, the combination of YAP1 and TAZ analysis by IHC may be useful in $\mathrm{MM}$ diagnosis.

The positive staining rates for YAP1 and TAZ in epithelioid, biphasic and sarcomatoid MM are presented in Fig. 4. The expression of YAP1 was significantly lower in sarcomatoid compared with the epithelioid and biphasic types $(\mathrm{P}=0.0003)$.

\section{Discussion}

Malignant mesothelioma is an aggressive tumor and the number of patients with MM is expected to increase worldwide in the future (4). Accurate and early pathological diagnosis of MM may improve patient outcomes, as patients with early MM 

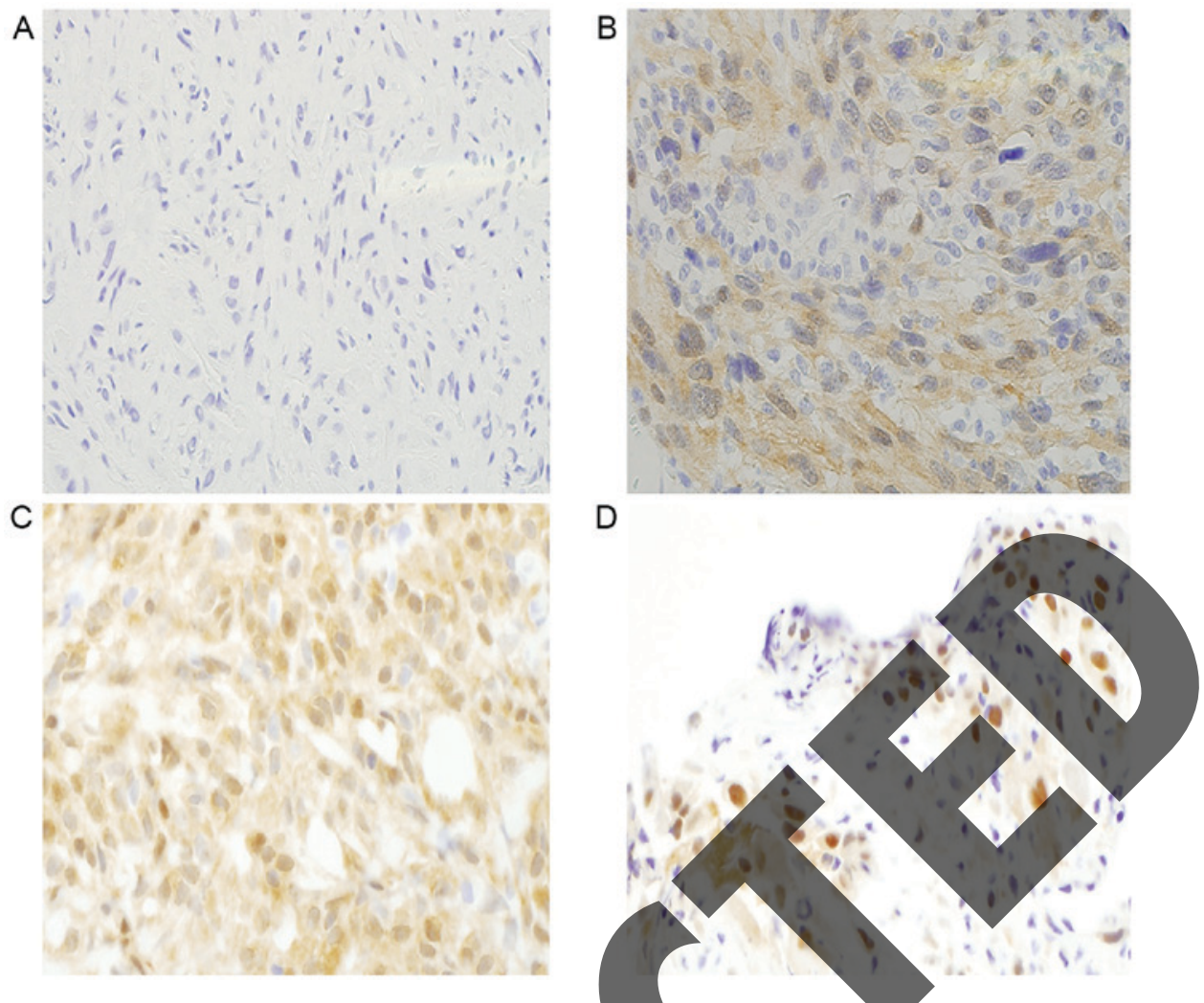

Figure 1. Representative images of immunohistochemical analysis results for YAP1 (magnification, x400). (A) Negative YAP1 staining (score, 0). (B) Weaker nuclear staining compared with that in the cytoplasm (score, 1+). (C) Nuclear staining equivalent compared with that in the cytoplasm (score, 2+). (D) Stronger nuclear staining compared with that in the cytoplasm (score, 3+). Scores of 2+or 3+ were considered to indicate YAP1-positive staining. YAP1, yes-associated protein 1.

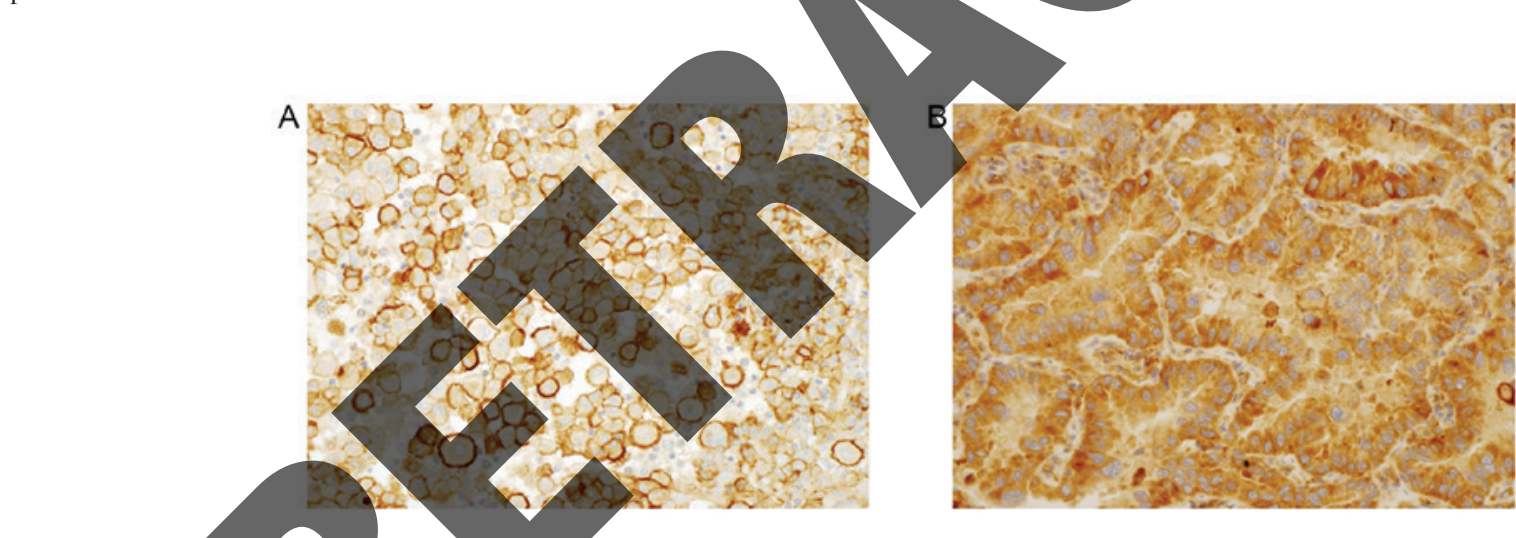

Figure 2. Representative images of immunohistochemical analysis results for TAZ (x400). (A) Strong staining in the cell membrane was considered a TAZ-positive result. (B) Nonmembranous staining was disregarded. TAZ, tafazzin.

may be eligible for multimodal therapy, including surgery. Therefore, IHC analysis is important, and several biomarkers have been evaluated for their utility in diagnosing MM. Sheffield et al (5) and Minato et al (1) identified numerous markers detectable by IHC and FISH for the diagnosis of MM. In previous studies, p16 homozygous deletion and loss of $B A P 1$ were not detected by FISH and IHC, respectively, in benign mesothelial proliferations; this result suggests that the identification of p16 homozygous deletion by FISH and loss of BAPI by IHC may be useful for distinguishing benign tumors from malignant tumors $(4,5,15,16)$. However, despite the high specificity of p16 homozygous deletion and loss of $B A P 1$, their sensitivity was low.

Asbestos-exposed NF2 knockout mice exhibit accelerated MM tumor formation; therefore, it is possible that the inactivation of $N F 2$ is important in the development of MM $(2,17)$. The Hippo pathway, which is induced by $N F 2$, exhibits cross-talk with important pathways, including the transforming growth factor $\beta$ /bone morphogenetic protein pathway and Wnt pathway, for the development and progression of malignant tumors $(2,11,18)$. The Hippo pathway regulates YAP1/TAZ. In addition, cell junction proteins, mechanical stretch and certain tumor-development pathways also regulate YAP1/TAZ via interaction with various transcriptional factors. In addition, TAZ is associated with the differentiation of mesenchymal cells; the expression of TAZ is increased following epithelial-mesenchymal transition (19). Staining of TAZ in the cell membrane occurred in a high proportion of MM cells, including those of sarcomatoid-type MM in the present study. The intracellular 
Table II. Results of the immunohistochemical analysis of YAP1 and TAZ in MM cells and RMCs.

\begin{tabular}{lccc}
\hline Type & Total patients, n & $\begin{array}{r}\text { YAP1-positive } \\
\text { patients, n (\%) }\end{array}$ & $\begin{array}{r}\text { TAZ-positive } \\
\text { patients, n (\%) }\end{array}$ \\
\hline MM & 31 & $27(87)$ & $28(90)$ \\
RMC & 33 & $15(45)$ & $18(55)$ \\
\hline
\end{tabular}

YAP1, yes-associated protein 1; TAZ, tafazzin; MM, malignant mesothelioma; RMC, reactive mesothelial cell.

Table III. Sensitivity and specificity of immunohistochemical analysis of YAP1, TAZ, and the combination of YAP1 and TAZ for the differential diagnosis of MM from RMC when the cutoff points were set at 6 for YAP1 and at 3 for TAZ.

\begin{tabular}{lccc}
\hline Parameter & YAP1 & TAZ & YAP1 and TAZ \\
\hline MM, n/total & $26 / 31$ & $27 / 31$ & $23 / 31$ \\
RMC, n/total & $7 / 33$ & $13 / 33$ & $2 / 33$ \\
Sensitivity, \% & $84(71-97)$ & $87(75-99)$ & $74(59-89)$ \\
$(95 \%$ CI) & & & \\
Specificity, \% & $79(65-93)$ & $61(44-78)$ & $94(86-100)$ \\
$(95 \%$ CI $)$ & & &
\end{tabular}

YAP1, yes-associated protein 1; TAZ, tafazzin; MM, malignant mesethelioma; RMC, reactive mesothelial cells; CI, confidence interval

localization of TAZ may differ between epithelial and meso thelial cells $(10,12,19)$. An alternative hypothesis is that the difference in staining sites of YAP1 and TAZ may be caused by the difference in the clone used $(14,20,21)$. For the IHC of YAP1 and TAZ, a standard antibody clone has not yet been determined. The clone used may affect the site and intensity of staining.

In a previous investigation of the different histological subtypes of MM, the expression of $\mathrm{U} 3$ small nucleolar ribonucleoprotein and glucose transporter 1 tended to be higher in sarcomatoid MM(1). In addition, Takeda et al (4) and Illei et al (22) suggested that p16 homozygous deletion, detected by FISH, was more common in sarcomatoid MM compared with epithelioid MM. However, the loss of BAPI was more common in epithelioid MM compared with sarcomatoid MM $(23,24)$. The current study confirmed that the expression of YAP1 was higher in epithelioid and biphasic MM compared with sarcomatoid MM. However, the expression of TAZ was higher in sarcomatoid MM compared with YAP1. These results support the hypothesis that YAP1 and TAZ have different roles. Additionally, $N F 2$ gene mutations are involved in an alternative pathway that differ from p16 and $B A P 1$, thus these markers may aid in distinguishing MM from RMC.

For the first time, the present study demonstrated the expression of YAP1 and TAZ in MM and RMC using IHC, and examined them as potential markers of MM in clinical specimens. Notably, YAP1 and TAZ were found to

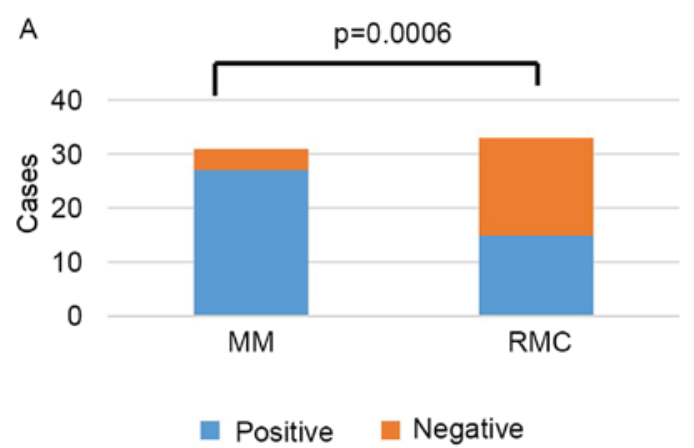

B
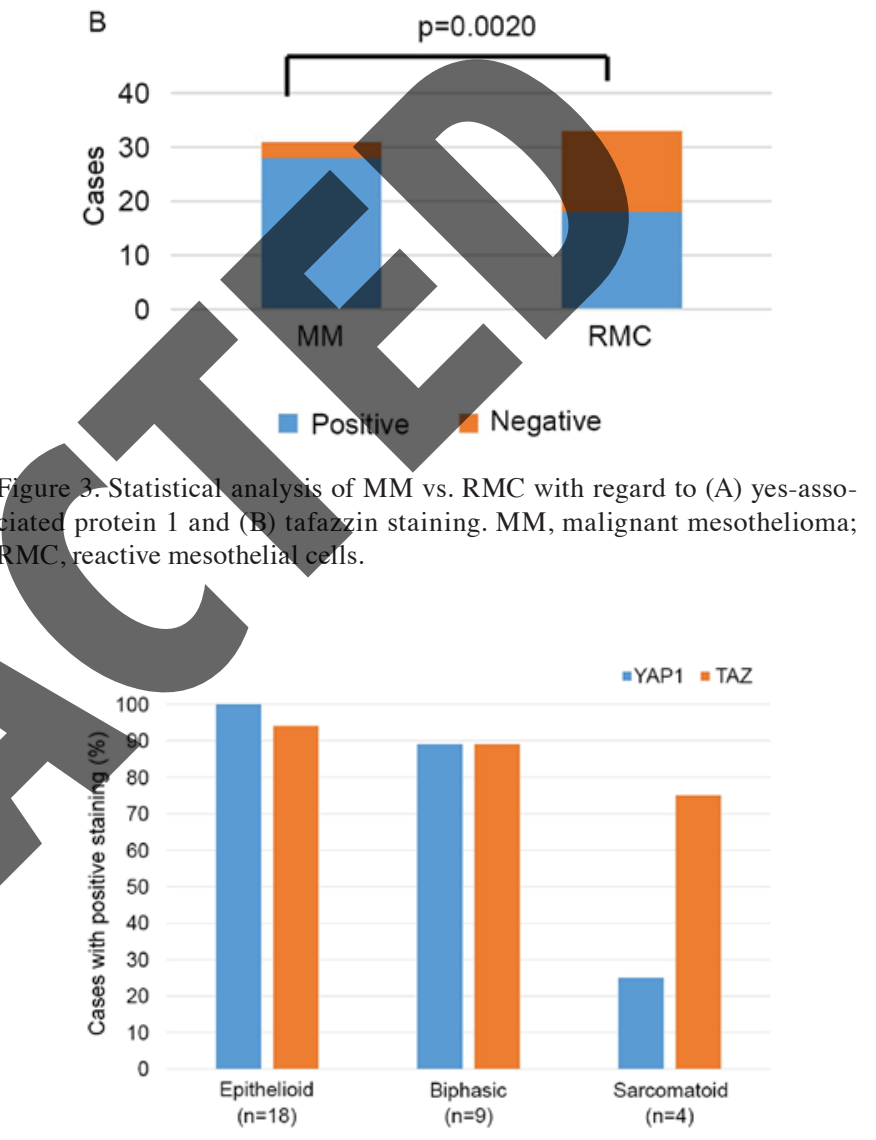

Figure 4. Expression of YAP1 and TAZ according to subtypes of malignant mesothelioma. Expression of YAP1 was significantly lower in sarcomatoid compared with epithelioid and biphasic types $(\mathrm{P}=0.0003)$. YAP1, yes-associated protein 1; TAZ, tafazzin.

be significantly more highly expressed in MM compared with RMC. In addition, the combination of YAP1 and TAZ staining was determined to have a sensitivity and specificity of 74 and $94 \%$, respectively, indicating that these markers combined may be helpful for distinguishing MM from RMC.

In summary, the present study confirmed that YAP1 and TAZ were more highly expressed in MM compared with RMC. These markers may helpful for distinguishing MM from RMC. Additional studies on a larger cohort of patients with $\mathrm{MM}$ are required to evaluate the utility and efficiency of this diagnostic approach.

\section{Competing interests}

The authors declare that they have no competing interests. 


\section{References}

1. Minato H, Kurose N, Fukushima M, Nojima T, Usuda K, Sagawa M, Sakuma T, Ooi A, Matsumoto I, Oda M, et al: Comparative immunohistochemical analysis of IMP3, GLUT1, EMA, CD146, and desmin for distinguishing malignant mesothelioma from reactive mesothelial cells. Am J Clin Pathol 141; 85-93, 2014.

2. Hata Y, et al: Journal of Clinical and Experimental Medicine 251: 351-356, 423-435, 2014. (In Japanese).

3. Bueno R, Stawiski EW, Goldstein LD, Durinck S, De Rienzo A, Modrusan Z, Gnad F, Nguyen TT, Jaiswal BS, Chirieac LR, et al: Comprehensive genomic analysis of malignant pleural mesothelioma identifies recurrent mutations, gene fusions and splicing alterations. Nat Genet 48: 407-416, 2016.

4. Takeda M, Kasai T, Enomoto Y, Takano M, Morita K, Kadota E and Nonomura A: 9p21 Deletion in the diagnosis of malignant mesothelioma, using fluorescence in situ hybridization analysis. Pathol Int 60: 395-399, 2010.

5. Sheffield BS, Hwang HC, Lee AF, Thompson K, Rodriguez S, Tse $\mathrm{CH}$, Gown AM and Churg A: BAP1 immunohistochemistry and p16 FISH to separate benign from malignant mesothelial proliferations. Am J Surg Pathol 39: 977-982, 2015.

6. Sekido Y: Molecular pathogenesis of malignant mesothelioma. Carcinogenesis 34: 1413-1419, 2013

7. Yokoyama T, Osada H, Murakami H, Tatematsu Y, Taniguchi T, Kondo Y, Yatabe Y, Hasegawa Y, Shimokata K, Horio Y, et al: YAP1 is involved in mesothelioma development and negatively regulated by Merlin through phosphorylation. Carcinogenesis 29: 2139-2146, 2008.

8. Murakami H, Mizuno T, Taniguchi T, Fujii M, Ishiguro F, Fukui T, Akatsuka S, Horio Y, Hida T, Kondo Y, et al: LATS2 is a tumor suppressor gene of malignant mesothelioma. Cancer Res 71: 873-883, 2011.

9. Cong W, Hirose T, Harita Y, Yamashita A, Mizuno K, Hirano H and Ohno S: ASPP2 regulates epithelial cell polarity through the PAR complex. Curr Biol 1408-1414, 2010.

10. Liu CY, Lv X, Li T, Xu Y, Zhou X, Zhao S, Xiong Y, Lei QY and Guan KL: PP1 cooperates with ASPP2 to dephosphorylate and activate TAZ. J Biol Chem 286: 5558-5566, 2011.

11. Grannas K, Arngården L, Lönn P, Mazurkiewicz M, Blokzijl A Zieba A and Söderberg O: Crosstalk between Hippo and TGFb Subcellular localization of YAP/TAZ/Smad complexes. J Mol Biol 427: 3407-3415, 2015.

12. Wells CD, Fawcett JP, Traweger A, Yamanaka Y, Goudreault M Elder K, Kulkarni S, Gish G, Virag C, Lim C, et al:A Rich1/Amo Complex Regulates the Cdc42 GTPase and apical-polarity proteins in epithelial cells. Cell 125: 535-548, 2006.

13. Travis WD, Brambilla E, Burke AP, Marx A and Nicholson AG (eds): WHO Classification of Tumours of the Lung, Pleura, Thymus and Heart. 4th edition, WHO, Geneva, pp154-171, 2015.
14. Yue G, Sun X, Gimenez-Capitan A, Shen J, Yu L, Teixido C, Guan W, Rosell R, Liu B and Wei J: TAZ is highly expressed in gastric signet ring cell carcinoma. Biomed Res Int 2014: 393064 , 2014.

15. Chiosea S, Krasinskas A, Cagle PT, Mitchell KA, Zander DS and Dacic S: Diagnostic importance of 9p21 homozygous deletion in malignant mesithliomas. Mod Pathol 21: 742-747, 2008

16. Hwang HC, Sheffield BS, Rodriguez S, Thompson K, Tse $\mathrm{CH}$, Gown AM and Churg A: Utility of BAP1 immunohistochemistry and p16 (CDKN2A) FISH in the diagnosis of malignant mesothelioma in effusion cytology specimens. Am J Surg Pathol 40: 120-126, 2016.

17. Altomare DA, Vaslet CA, Skele KL, De Rienzo A, Devarajan K, Jhanwar SC, McClatchey AI, Kane AB and Testa JR: A mouse model recapitulating molecular features of human mesothelioma. Cancer Res 65: 8090-8095, 2005.

18. Fujii M, Toyoda T, Nakanishi H, Yatabe Y, Sato A, Matsudaira Y, Ito $\mathrm{H}$, Murakami $\mathrm{H}$, Kondo Y, Kondo E, et al: TGF-b synergizes with defects in the Hippo pathway to stimulate human malignant mesothelioma growth. J Exp Med 209: 479-494, 2012.

19. Cordenonsi M, Zanconato F, Azzolin L, Forcato M, Rosato A, Frasson C, Inui M, Montagner M. Parenti AR, Poletti A, et al: The Hippo transducer TAZ confers cancer stem cell-related traits on breast cancer cells Cell 147: 759-772, 2011.

20. Xie M, Zhang L, He CS, Hou JH, Lin SX, Hu ZH, Xu F and Zhao HY: Prognostic significance of TAZ expression in resected non-small cell lung cancer. J Thorac Oncol 7: 799-807, 2012

21. Li PD, Wang XJ, Shan Q, Wu YH and Wang Z: Evaluation of $\mathrm{TAZ}$ expression and its effect on tumor invasion and metastasis in human glioma. Asian Pac J Trop Med 7: 757-760, 2014.

Illei PB, Rusch VW, Zakowski MF and Ladanyi M: Homozygous deletion of CDKN2A and codeletion of the methylthioadenosine phosphorylase gene in the majority of pleural mesotheliomas. Clin Cancer Res 9: 2108-2113, 2003.

23. Singhi AD, Krasinskas AM, Choudry HA, Bartlett DL, Pingpank JF, Zeh HJ, Luvison A, Fuhrer K, Bahary N, Seethała RR and Dacic S: The prognostic significance of BAP1, NF2, and CDKN2A in malignant peritoneal mesothelioma. Mod Pathol 29: 14-24, 2016.

Carbone M, Shimizu D, Napolitano A, Tanji M, Pass HI, Yang H and Pastorino S: Positive nuclear BAP1 immunostaining helps differentiate non-small cell lung carcinomas from malignant mesothelioma. Oncotarget 7: 59314-59321, 2016.

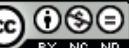

This work is licensed under a Creative Commons Attribution-NonCommercial-NoDerivatives 4.0 International (CC BY-NC-ND 4.0) License.

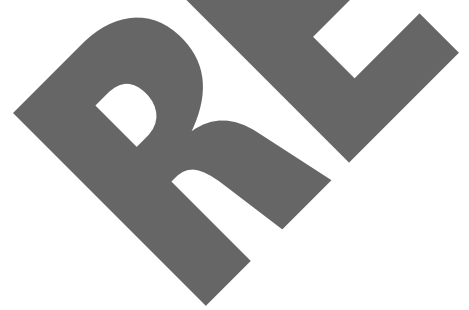

